\title{
Fast geophysical prospecting applied to archaeology: results at «Villa ai Cavallacci» (Albano Laziale, Rome) site
}

\author{
Stefano Urbini $\left({ }^{1}\right)$, Lili Cafarella $\left({ }^{1}\right)$, Marco Marchetti $\left({ }^{1}\right)$, Pino Chiarucci $\left({ }^{2}\right)$ and Davide Bonini $\left({ }^{3}\right)$ \\ (1) Istituto Nazionale di Geofisica e Vulcanologia, Roma, Italy \\ ${ }^{(2)}$ Museo Civico di Albano Laziale, Albano Laziale (RM), Italy \\ (3) Dipartimento per il Territorio e le sue Risorse (DipTeRis), Università degli Studi di Genova, Italy
}

\begin{abstract}
The present essay is the result of a cooperative work between geophysicists and archaeologists in which the authors carried out an integrated geophysical prospecting in an archaeological site near Rome. This paper describes the methodology and the results of a geophysical survey carried out on Villa ai Cavallacci, an ancient roman building in Albano Laziale (Rome) discovered in the late seventies. It is often possible to obtain very important results planning a fast geophysical survey opportunely; within this framework (due to the fact that an archaeological excavation was planned in a short time), an integrated geophysical techniques survey (GPR, magnetic, and geoelectric tomography) has been carried out on the areas indicated by the archaeologists. Even if the described geophysical survey should be considered only a first step analysis, the data pointed out some very interesting features confirmed by the excavation.
\end{abstract}

Key words archaeology - integrated geophysical prospecting - GPR - geoelectric tomography - magnetic survey

\section{Introduction}

It is well known that, especially in archaeology, economic and human resources are scant. Digging is expensive, takes a long time and sometimes is also unfruitful. Geophysical surveying provides a relatively fast, non invasive and low cost tool that succeeds in obtaining different kinds of information on shallow subsurface features. The purpose of the geophysical survey is, in fact, to investigate the site response

Mailing address: Dr. Stefano Urbini, Istituto Nazionale di Geofisica e Vulcanologia, Via di Vigna Murata 605, 00143 Roma, Italy; e-mail: urbinis@ingv.it to different geophysical prospecting, to define the position of interesting structures that can be investigated by archaeologists.

In the case of Villa ai Cavallacci sited in Albano Laziale (Rome) and reported in fig. 1, the authors carried out an acquisition test using different geophysical methods, focused to obtain a high-resolution data set. An archaeological excavation (performed in September 2005) carried out according to what had been previously pointed out by geophysics, yielded many ruins. In particular an ancient room with a collapsed roof was revealed, confirming the importance of the cooperation between the expertises of both archaeology and geophysics.

\section{Site archeological characteristics}

In Republican and Imperial times, a number of famous Romans built country houses and vil- 


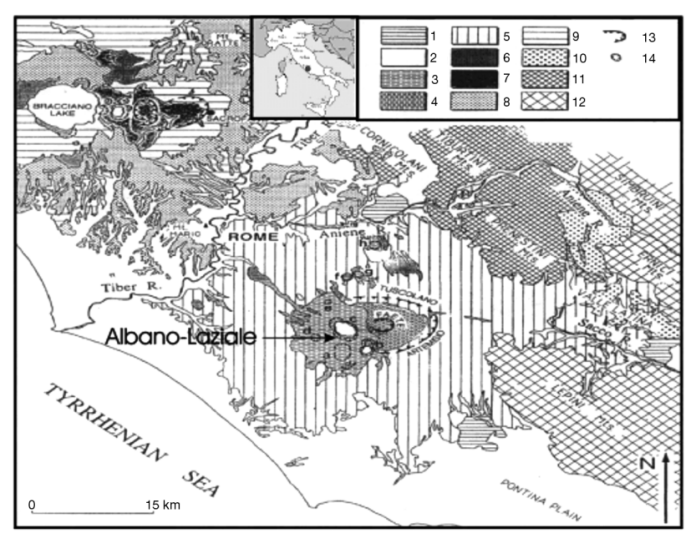

Fig. 1. Location of the survey site.

las in the Alban Hills, especially in the Albano Laziale town area. Some of these were great luxury buildings, such as the Roman villa discovered in the Cavallacci area, in Via Verdi. The villa probably belonged to a member of the imperial family because, during excavations, a large quantity of fine-quality marble emerged. The Villa ai Cavallacci was discovered in 1975 but the archaeological investigation (performed by the Civic Museum of Albano Laziale) started only ten years later, in 1986 and is still in progress. Only a small part of the archaeological site was excavated and the aim of the geophysical survey was to realize a spatial reconstruction of shallow buried walls and rooms, to define new possible excavation sites. The digging has revealed many masonry structures, rooms with mosaic and polychrome marble floors. The structure, which was built towards the end of the republican period, reached the height of its magnificence during the Tiberius era and in III century A.D. under Severus age. It was inhabited until V century A.D. (Chiarucci, 1990). Starting from the end of the III century A.D. the building was used only as a burial place until the residential building, already neglected and quit, became a country villa.

\section{Geophysical methods}

The primary goal of this survey was the identification of targets of potential interest in an area partially investigated by archaeologists in previous excavations. To obtain high resolution results in a short time, a preliminary magnetic analysis and a large step GPR grid were carried out on the areas indicated by the archaeologists. Then some new GPR and geoelectrical profiles were performed to obtain new details on the most interesting areas identified by the preliminary investigation.

\subsection{GPR survey}

The GPR method is based on the reflection of an electromagnetic wave due to one or more discontinuities in the media dielectric properties. The main physical factor that can generate a reflected radar wave is the dielectric contrast between different media. Buried shallow walls and holes generate a good contrast in the field of dielectric properties providing a possible and resolvable target for the GPR method (Annan and Cosway, 1992; Jol and Smith, 1992; Benson, 1995; Daniels, 1996; Basile et al., 2000).

Analysing the direct waves on a WARR test dataset produced a value of about 5.7 for the dielectric constant of the surrounding medium (a silty sand) using an averaged electromagnetic wave speed of about $0.125 \mathrm{~m} / \mathrm{ns}$. The buried structures were essentially made of limestone blocks and marble floors; both showed a good dielectric contrast from surrounding medium properties.

GPR profiles were mainly carried out along NW and SW directions, as shown in fig. 2, by GSSI Sir10B instrumentation equipped with a $400 \mathrm{MHz}$ monostatic antenna.

Unfortunately, many anthropic obstacles (excavation scraps, power lines, etc.) hold up a regular spaced acquisition greed. For this reason, we employed a two step profiling method. First, lines were acquired $3 \mathrm{~m}$ spaced and then, according to the results of the recorded GPR profiles, they were thickened as much as possible only where it was needed.

The acquired profiles showed many archaeologically interesting anomalies. Figure 3 shows the position of some of them by A, B and C boxes, where red and green indicate anomalies linked to buried walls and flat high energy reflec- 
Wooden fence

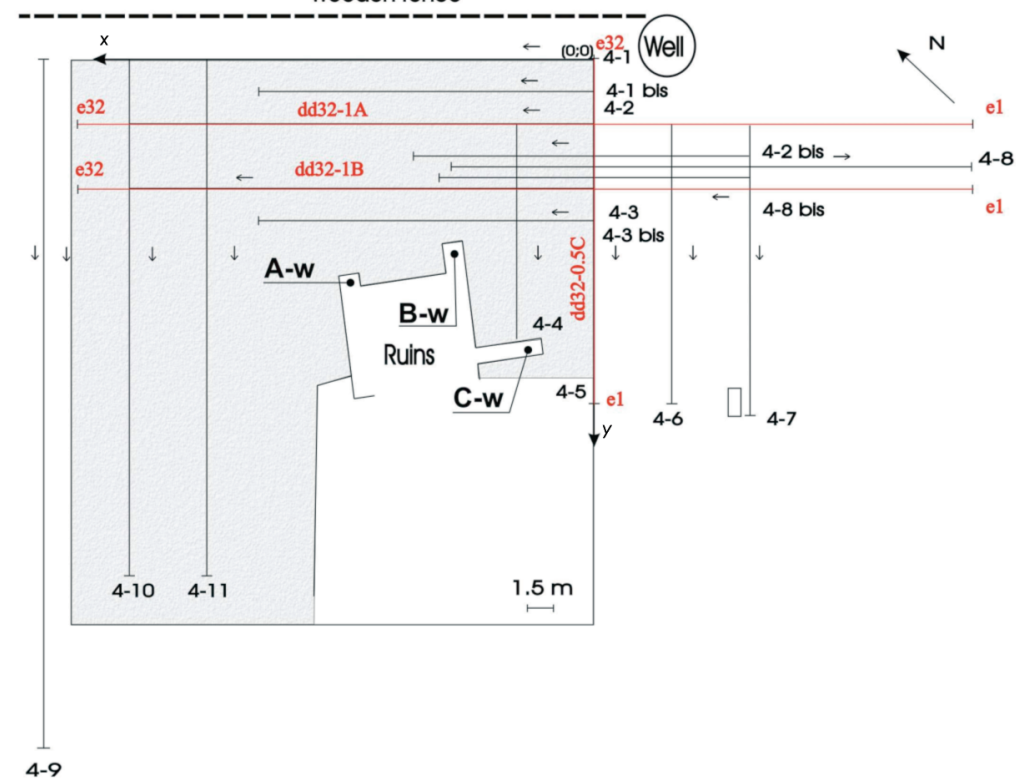

Fig. 2. Acquisition scheme: GPR profile (black lines); geoelectric tomography (red lines); magnetic survey (gray area).

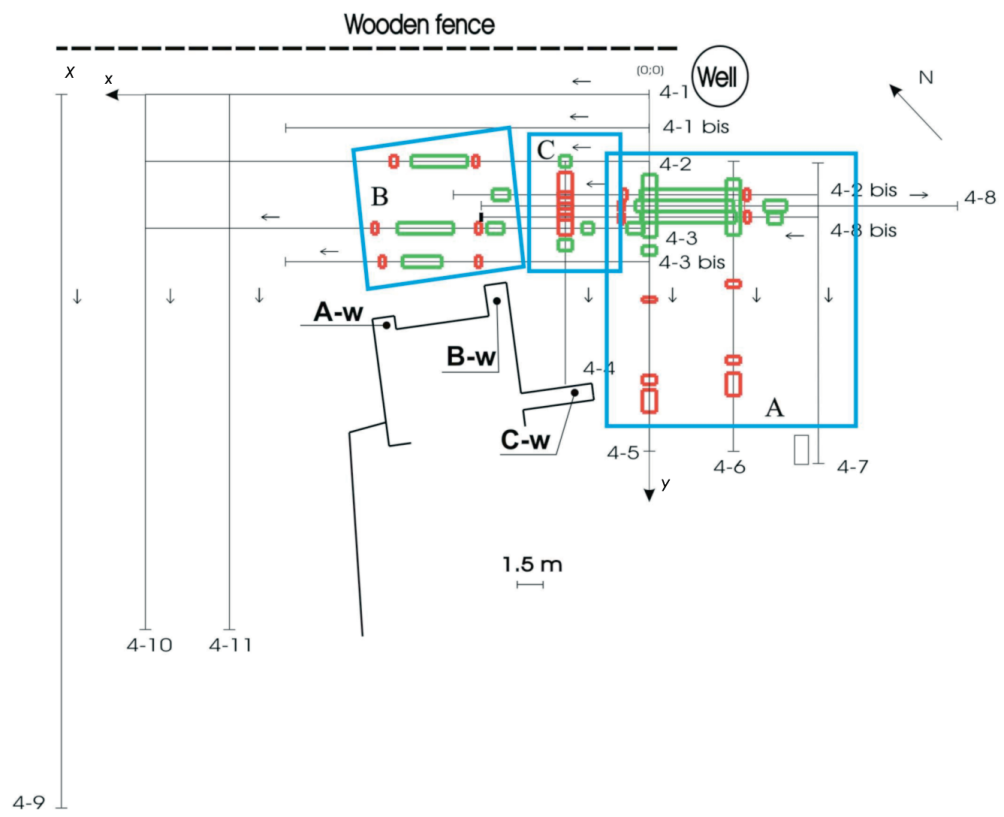

Fig. 3. Structures pointed out by the GPR investigation. 
tors respectively. The structures located in area B seem to be the extension of the excavated and visible ruins, while anomalies in the upper part of $\mathrm{A}$ and $\mathrm{C}$ areas could be linked to the same structure. Figure 4 reports an example of one of these radar profiles (Alb 4.8): yellow boxes identify anomalies that show shape, energy and alignment suggesting they could be generated by buried walls $35-40 \mathrm{~cm}$ wide. In the same figure the flat high-energy reflection in the blue box (occurred at $29 \mathrm{~ns}$, from 5 to $9 \mathrm{~m}$ along the line) could be generated by a buried floor. Right above this reflection, as shown in the figure, electromagnetic wave propagation changes rapidly probably because of a different kind of filling material between the two walls (probably filling full of air). Looking at the local excavated ruins and from a digging test, the authors' interpretation was oriented towards a room with a collapsed roof. In order to verify this result, additional GPR lines were carried out on the more interesting zones. Then the reflections from two perpendicular lines (Alb 4.8 and Alb 4.5 reported in fig. 5) were compared. With a radar wave speed of $0.125 \mathrm{~m} / \mathrm{ns}$, both radar profiles pointed out a continuous flat reflection $1.8 \mathrm{~m}$ deep. Also the previously identified filling area was clearly observed along both directions.
Finally a 3D slices reconstruction was attempted. Figure 6 overlaps the time slices located at a depth of $0.5 \mathrm{~m}$ on the map confirming previous results.

\subsection{Geoelectric tomography}

Geoelectrical tomography on archaeological sites indicates spatial differences in sediment moistures: the presence of features like architecture, activity areas and archaeological remains can be detected if the amount of moisture they retain is different from the surrounding sediment (Bernabini et al., 1988; Brizzolari et al., 1992; Pellerin and Wannamaker, 2005). This technique allows a picture of the electrical properties of the subsurface to be built by passing an electrical current along different paths and measuring the associated voltages. Multi-electrode instrumentation permits several resistivity measurements to be made with different methods at the same time (Jordan and Costantini, 1999). This work used only a dipole-dipole configuration. The dipole-dipole array is very sensitive to horizontal changes in resistivity, so it can be used to map vertical structures such as dykes and cavities. This investigation made three profiles using a resistivity

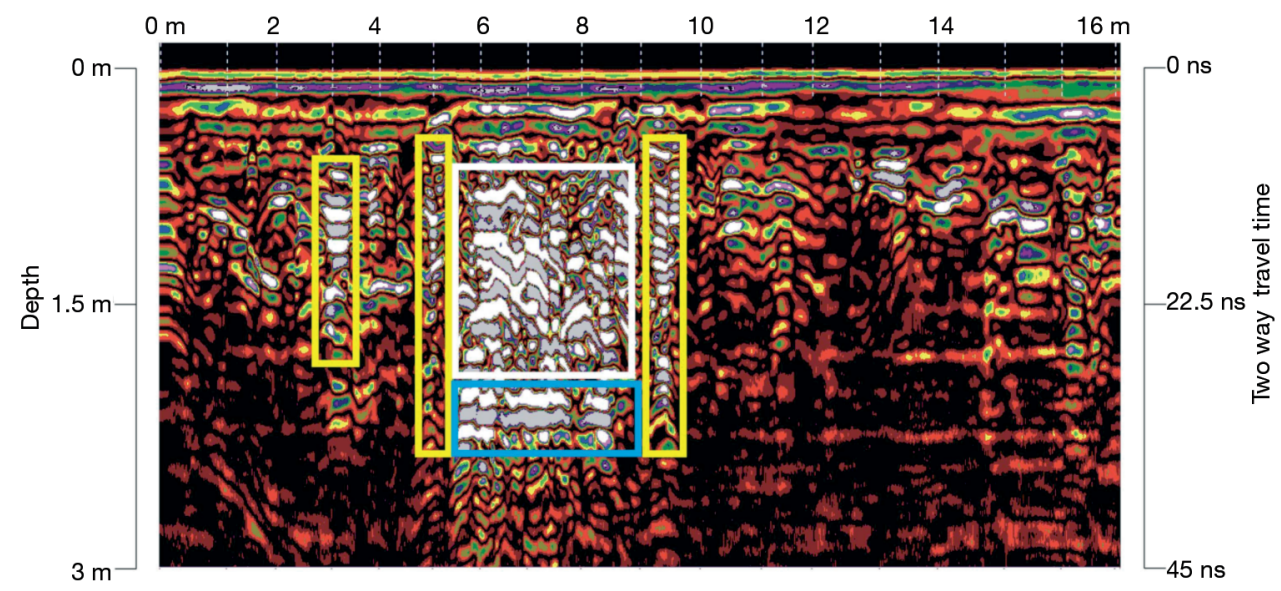

Fig. 4. Migrated and stacked GPR section Alb 4.8 (see fig. 3 for line position). Yellow boxes indicate anomalies due to wall response while blue box indicates a flat high-energy reflection probably due to a buried floor. The white box indicates a room filled up with backfill. 


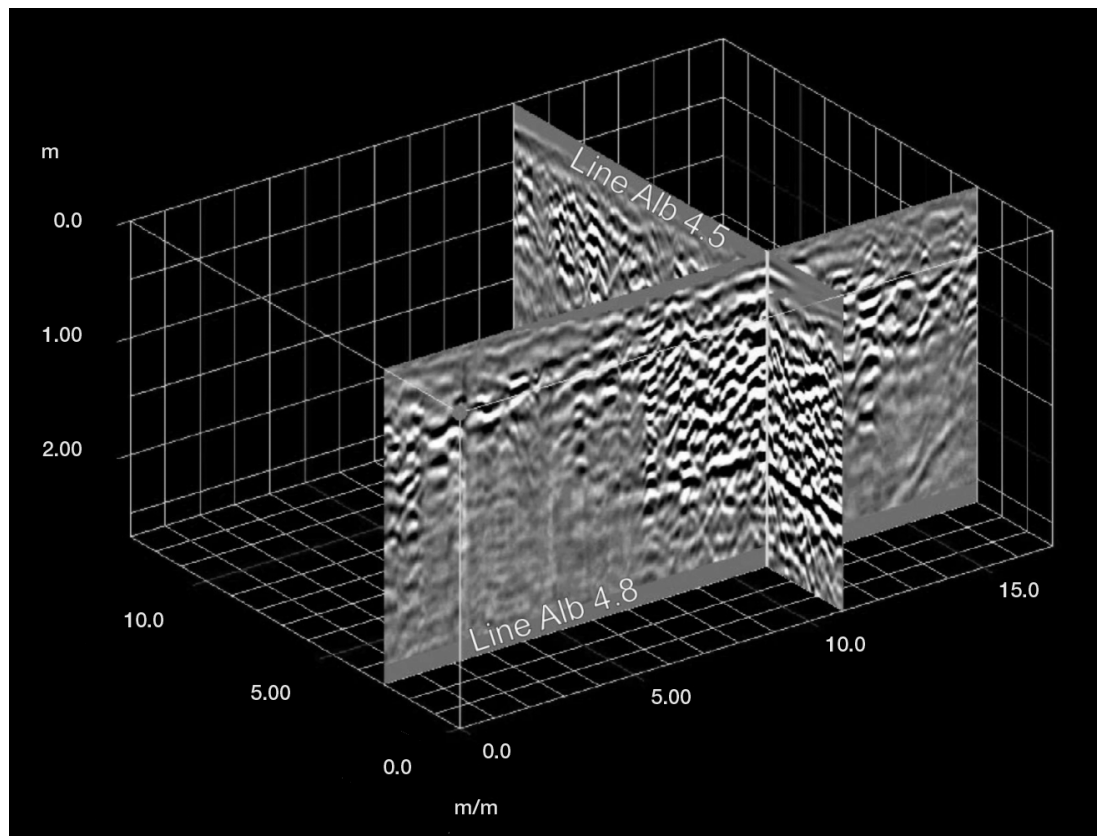

Fig. 5. Perpendicular GPR lines Alb 4.8 and Alb 4.5 plot showed the $2 \mathrm{D}$ continuity of anomaly due to the buried room.

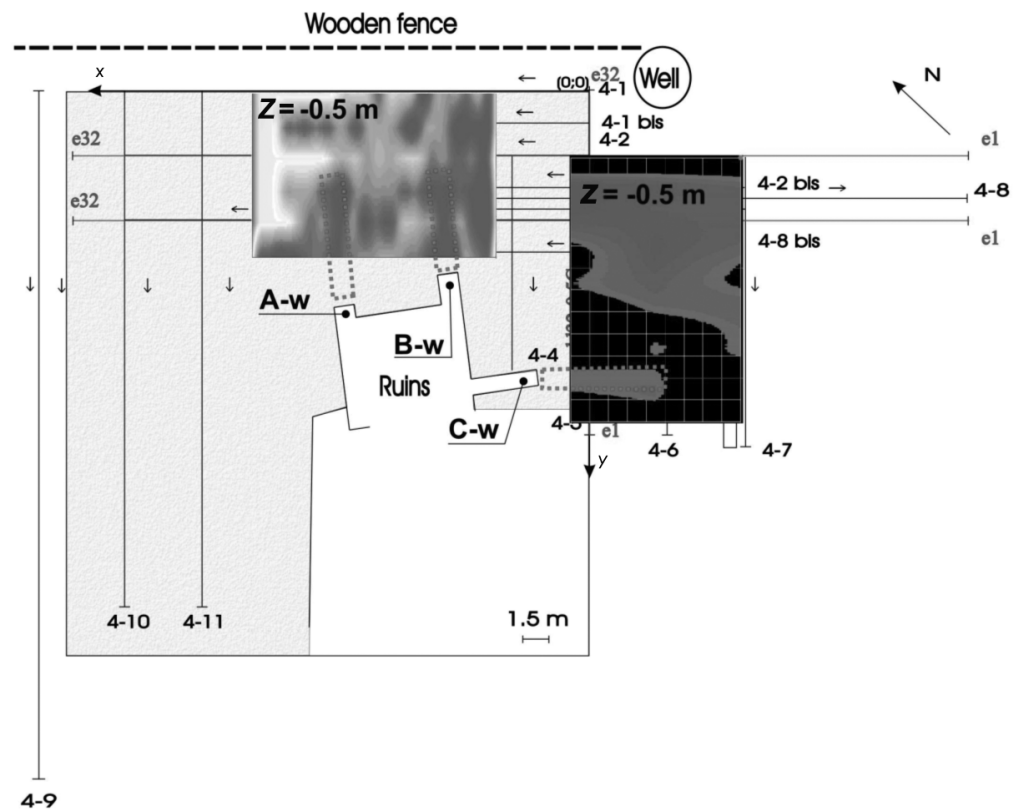

Fig. 6. Overlap between two $0.5 \mathrm{~m}$ depth $3 \mathrm{D}$ GPR model slices and the site map. 
meter Iris-Syscal R2. The profiles were carried out by means of dipole-dipole electrode arrays, using electrodes with $1 \mathrm{~m}$ (the two $\mathrm{N}-\mathrm{W}$ profiles) and $0.5 \mathrm{~m}$ (for the $\mathrm{S}-\mathrm{W}$ ) spacing respectively. Also multi-electrode profiles were carried out to investigate the existence of the (interpreted) buried room pointed out by the GPR analysis. The three geoelectrical profiles were positioned according to the three most meaningful GPR lines. The selected electrode spacings were able to clearly detect the possible presence of a filled gap. Figure 7 reports the geoelectrical dd32-0.5C profile as an example. The searched filled gap is visible on the plot as a large red spot. The improved resolution of $0.5 \mathrm{~m}$ electrodes spacing also allowed the identification (in spite of its reduced depth information) of a wall structure $40-50 \mathrm{~cm}$ wide (same figure dotted line box). Geoelectric tomography confirms the GPR anomalies map and fig. 8 reports the perfect overlapping of resistivity and dielectric anomalies.

During September 2005 archaeologists performed a digging campaign and they followed the geophysics indications. Many results were confirmed, and in particular the identified room with a collapsed roof was revealed (see fig. 9).

\subsection{Magnetic survey}

Finally, a magnetic survey was performed on an area of about $440 \mathrm{~m}^{2}$. The aim of a magnetic survey is to reveal contrast in the magnetic property of the soils on the basis of anomalies induced in the Earth's magnetic field (Weymounth and Huggins, 1985). Magnetic method is usually used in archaeological exploration to detect features such as buried walls and structures, pottery, bricks, fire pits, buried pathways, tombs and numerous objects. The features are detected and mapped as a result of their being more magnetic than surrounding material (Patella, 1991).

This investigation collected magnetic data using an optically pumped caesium magnetometer Geometrics G858 in gradiometer configuration (two sensors mounted on a vertical
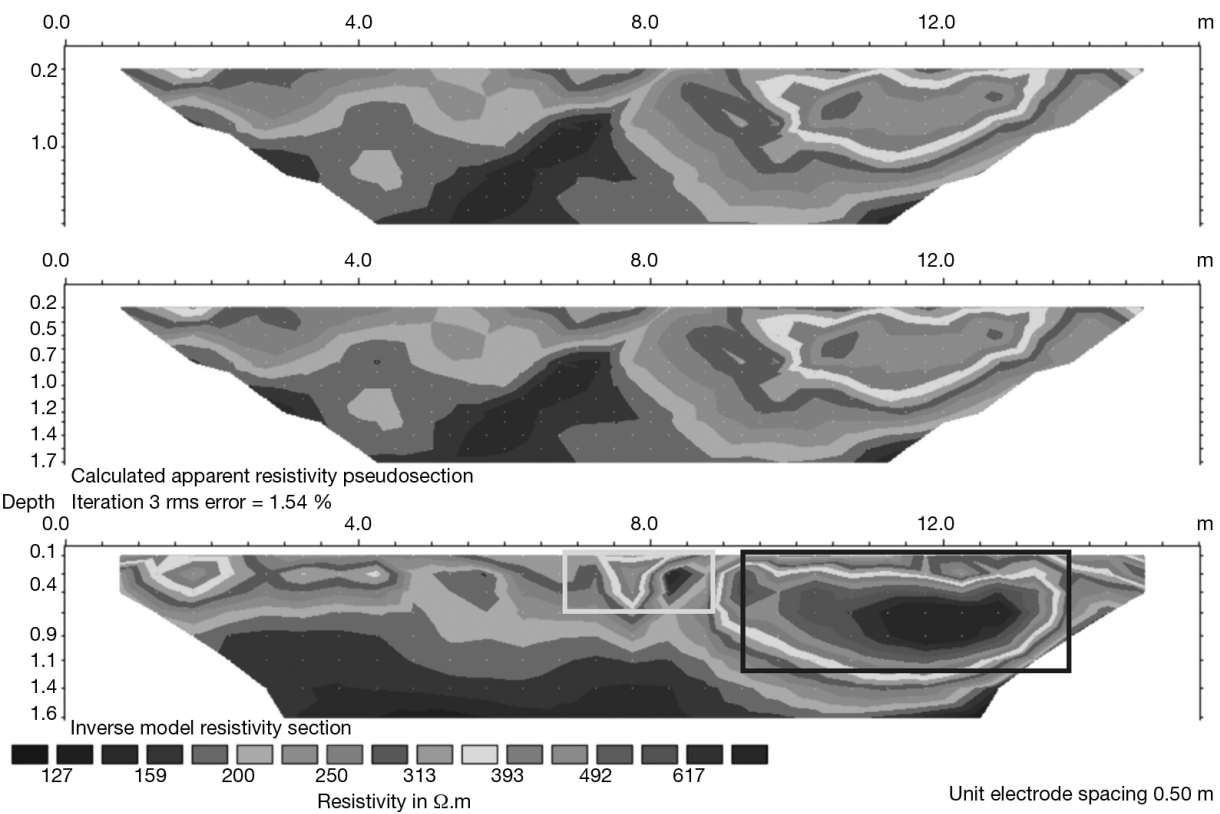

Fig. 7. Geoelectric multi-electrode dipole-dipole profile. Dotted line box identifies anomaly due to a wall structure. The white box shows the anomaly linked to the room. 


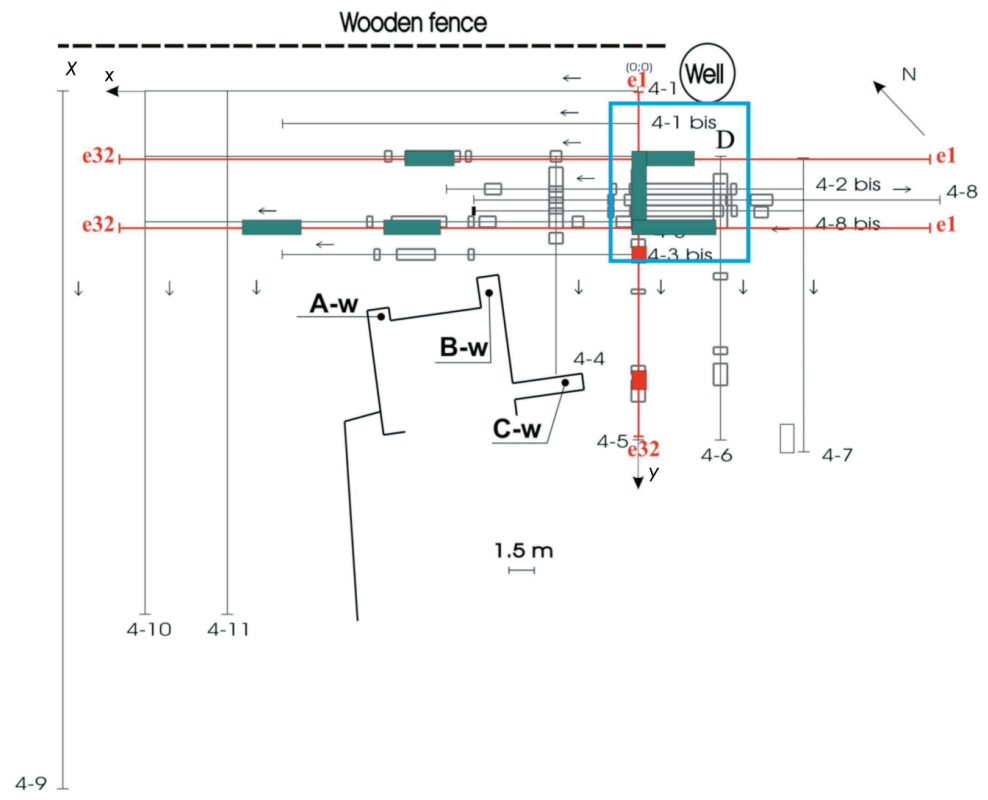

Fig. 8. Overlap between main GPR (gray) and geoelectrical anomalies.

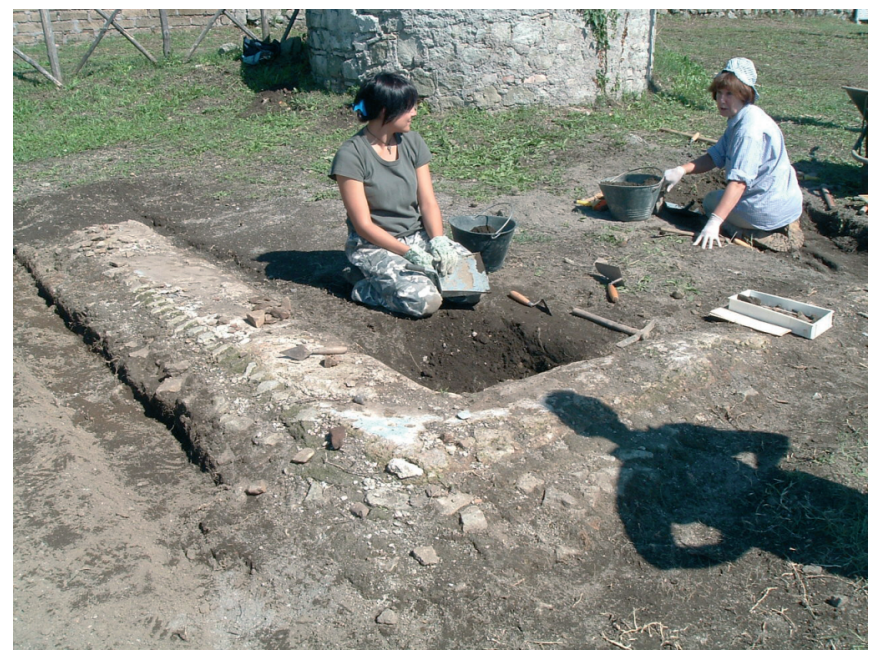

Fig. 9. Results of the September 2005 excavation. The wall of an ancient room are very evident in the picture. Following digging revealed that the room is filled with roofing material, as the authors presumed.

staff at a distance of $0.5 \mathrm{~m}$ apart). About 1500 measurements were made in 42 profiles spaced $0.5 \mathrm{~m}$ with a sampling step of $0.5 \mathrm{~m}$. In the studied site, the magnetic method was not very decisive. Magnetic measures were affected by low contrast in magnetic properties between structures and surrounding medium, recent human activities (agriculture for example), exca- 


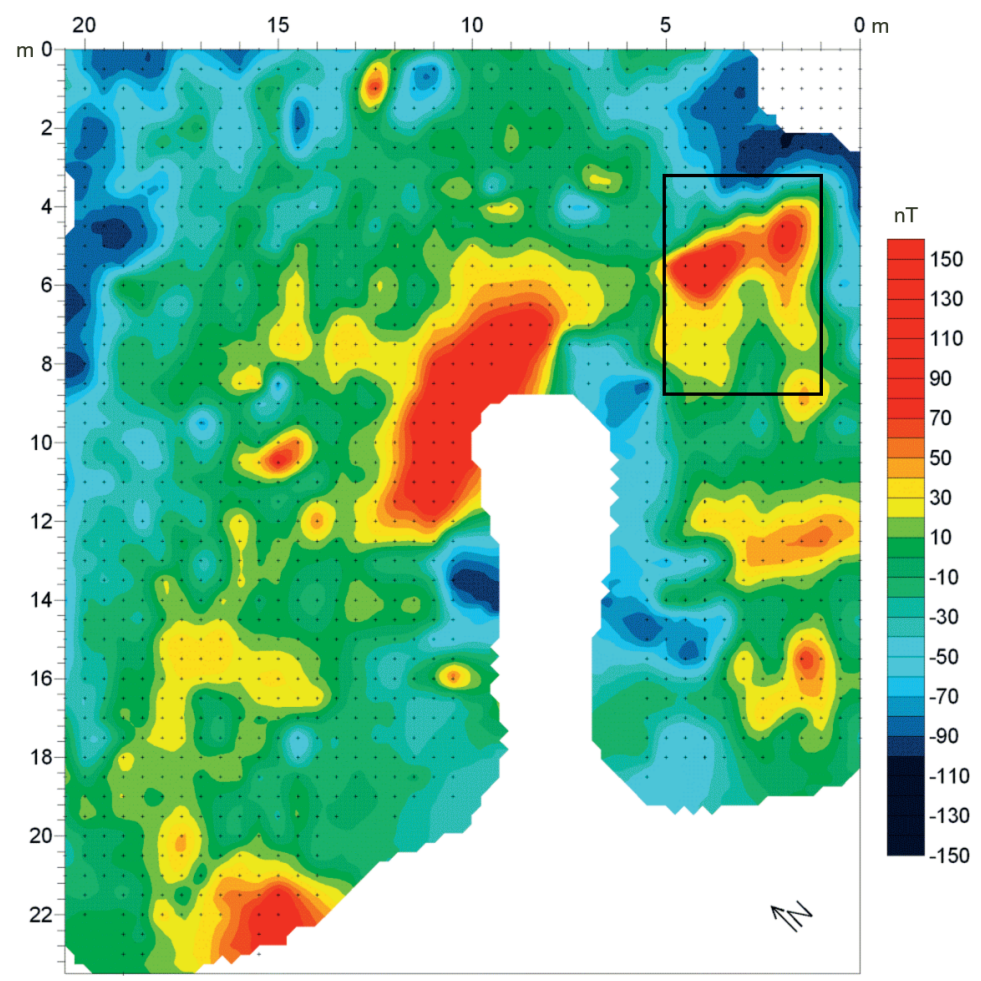

Fig. 10. Magnetic vertical gradient anomaly map.

vations and the presence of many abandoned steel materials. Therefore clear magnetic anomalies referable to the buried features are not very evident on the maps of the magnetic vertical gradient (fig. 10). The central part of the figure shows a large anomaly. It is linked to a steel sheet partially buried in the investigated area and conceals a possible anomaly related to the ruins both detected by the GPR and geoelectrical analysis. Only an anomaly (black box in fig. 10) is probably referable to buried structures (probably walls) also resulting from the GPR investigation (see fig. 3, box C).

\section{Conclusions}

This paper reports the results of an integrated geophysical survey performed in an archaeo- logical site in Albano Laziale, close to Rome. The investigated area was a Roman villa partially excavated by archaeologists in previous surveys. The aim of this survey was to define, in a few days, the presence and position of some structures that could be investigated in the planned archaeological excavation (September 2005). Test planning was focused to design a really fast but exhaustive measurement campaign. From this point of view, an integrated geophysical techniques survey (GPR, magnetic, and geoelectric tomography) was carried out on this archaeological site. Results from GPR and geoelectric tomography showed an unexpected good overlap. On the contrary, the magnetic survey result did not show clear magnetic anomalies referable to the buried features. In fact, the magnetic survey pointed out an unexpected poor quality result compared to the other methods 
probably because of the adverse application conditions (low magnetic contrast between backfill and remains, heavy human activities perturbation, small area investigated, abandoned steel materials, etc.). On the whole, the described test survey was able to detect and define the presence of some very interesting geophysical anomalies.

During September 2005 a digging campaign was conducted at the Roman Villa. Thanks to the results of the geophysical survey, the expected ancient buried room, well identified by GPR and geoelectric tomography, was excavated with other minor finds. These results confirmed, once more, that integration between geophysical techniques and archaeology is a really powerful tool. Also a simple acquisition test, if well planned, can turn into a meaningful survey.

\section{REFERENCES}

AnNAN, A.P. and S.W. Cosway (1992): Ground penetrating radar survey design, in Proceedings of the SAGEEP, 329-350.

Basile, V., M.T. Carrozzo, S. Negri, L. Nuzzo, T. QuarTA and A.V. VILlani (2000): A ground penetrating radar survey for archaeological investigations in an urban area (Lecce-Italy), J. Appl. Geophys., 44, 15-32.

Benson, A.K. (1995): Application of ground penetrating radar in assessing some geological hazards: example of groundwater contamination, faults, cavities, J. Appl. Geophys., 33, 177-193.

Bernabini, M., E. Brizzolari and S. Piro (1988): Improvement of signal-to-noise ratio in resistivity profiles, Geophys. Prospect., 36 (5), 559-570.

Brizzolari, E., F. Ermolli, L. Orlando, S. Piro and L. VERSINO (1992): Integrated geophysical methods in archaeological surveys, J. Appl. Geophys., 29 (1), 47-55.

Chiarucci, P. (1990): La villa di età romana ai «Cavallacci» in Albano, Quaderni del Centro di Studi per l'Archeologia Etrusco-Italica, 19 (10.2), 201-204.

DANIELS, D.J. (1996): Surface - Penetrating Radar (Institution of Electrical Engineers, London, U.K.), pp. 296.

JoL, H.M. and D.G. SMITH (1992): Ground penetrating radar: recent result, Can. Soc. Explor. Geophys. Rec., 27, 15-20.

JoRDAN, T. and D. Costantini (1999): The use of non-invasive electromagnetic (EM) tecniques for focusing environmental investigations, in Ground Conductivity Meters for Environmental Site Evaluation: Selected Papers and Examples (Geonics Ltd.), pp. 30.

PATElla, D. (1991): I principi della magnetometria e della gravimetria. Possibilità di applicazione in archeologia, in Proceedings of "Geofisica per l'Archeologia», Quaderni dell'Istituto per le Tecnologie Applicate ai Beni Culturali, 1, 71-81.

Pellerin, L. and P.E. Wannamaker (2005): Multi-dimensional electromagnetic modelling and inversion with application to near-surface Earth investigations, Comput. Electron. Agric., 46, 71-102.

Weymounth, J.W. and R. Huggins (1985): Geophysical surveying of archeological site, in Archeological Geol$o g y$, edited by G. RAPP and J.A. GIFFORD, 191-235.

(received October 10, 2006; accepted February 23, 2007) 